Journal of Social Sciences 6 (1): 29-32, 2010

ISSN 1549-3652

(C) 2010 Science Publications

\title{
The Model of Quality of Life Improvement for Chronic Patients in Community by Using Social Support
}

\author{
${ }^{1}$ Amorn Suwannimitr, ${ }^{2}$ Kanokjun Kamenkan, ${ }^{3}$ Choochart Deeromram, \\ ${ }^{3}$ Suwadee Jundeekrayom, ${ }^{4}$ Arun Leetong and ${ }^{5}$ Pornsawan Enkawan \\ ${ }^{1}$ Department of Community Nursing, \\ ${ }^{2}$ Department of Pediatric Nursing, \\ ${ }^{3}$ Department of Psychiatric Nursing, \\ Faculty of Nursing, Mahasarakham University, Thailand \\ ${ }^{4}$ Division of Thai Traditional Medicine, Cheang Yune Hospital, Mahasarakham Province, Thailand \\ ${ }^{5}$ Research Assistant, Mahasarakham University, Thailand
}

\begin{abstract}
Problem statement: Caring for a chronically ill-patients is a complex process which require the cooperation and social support to manage a chronic disease. It need an interaction of a large number of actors or collaborations from health care personnel of different organizations. Questions were raised to develop an appropriate intervention and the model of QOL improvement for chronicpatients in the community. Approach: To (1) develop the Quality Of Life (QOL) improvement for chronic illness patients in community by using social support program (2) evaluate the effects of the program on perception of illness, severity of illness, benefits and barriers of health promotion, health behaviors, Quality Of Life (QOL) and stress level. Participatory Action Research (PAR) was used. It was consisted of two phrases. The participants in first phase including with nurses, nutritionist, patients, caregivers, Village Health Volunteers (VHVs) and research team. The second phrase was to implement the interventions and evaluation. A total of $10 \mathrm{VHVs}$ and 50 participants who met the inclusion criteria. The intervention composed of 2 main programs; (1)The VHVs were trained for 1 month as a comprehensive program to be a healthcare team collaboration. (2)The chronically illpatients received main interventions including self-care education, apply Thai traditional medicine and home visits. Descriptive statistics and t-test were use to evaluate the pre-post intervention. Results: The majority of the participants were female $(n=38,76 \%)$, with the mean age of 66.68 years $(\mathrm{SD}=17.20)$, $85 \%$ caring by their children and $42.5 \%$ by their relatives. Most participants came from low income family (40\%). The post test score on each item showed that after intervention, changed scores on all five items (before-after), how ever the changes were statistically significantly $(<0.05)$ by some items, they were perceive of benefits and barriers of health promotion, health behaviors and stress level.. The rest as perceive of illness, perceive of severity of illness and QOL were not statistically significant $(>0.05)$. Conclusion: The findings suggested the set of interventions were effective to improve QOL of chronic patients and it was the appropriate model. To improve health care providers' knowledge, skills and increasing of collaborations among multidisciplinary team, Local Administration Organization (LAO). VHVs and Health Care Service Centre were strongly recommended to work with chronic patients.
\end{abstract}

Key words: Chronic patients, Quality of life, Social support

\section{INTRODUCTION}

Background and significance of research problem: Caring for a chronically ill-patients is a complex process, which requires the cooperation of all primary care professionals and their interaction with a large number of multi disciplines and health care personnel of different organizations (Panzarasaa et al., 2004). In addition, health care providers have sought to improve health outcomes through patient empowerment (Santurri, 2006). It was a challenge for both patient and family in preventing and managing chronic illness. It is 
also increasingly accepted that the public (as individuals, communities and the voluntary sector) should be involved in designing, delivering and evaluating services for chronic illness and in creating the conditions to support healthy living (Greenhalgh et al., 2009). Empowerment has been acknowledged as an alternative to compliance in order to guide the providerpatient relationship (Aujoulat et al., 2008).

Social support defined as the physical and emotional comfort that persons receive from their family, friends, co-workers and others. It is known that people were a part of a community that have other people who love and care. Social support is a concept that attempts to capture helping transactions that occur between people who share the same households, school, neighborhoods, workplaces, organizations and others community setting (Rappaport and Seidman, 2000).

Most chronic illnesses in Thailand, are diabetes mellitus and hypertension. These illnesses affect patients by limiting their quality of life. Mahasarakham province which is located in the north-eastern part of Thailand, ranked the first in patients with chronic illness according to the national survey (United Nations Development Programm, 2007/2008).

Cheang Yune village, a community with a population of 42,259. It has a community hospital which both serve as primary and secondary care as well as integrative Complementary Alternative Medicine (CAM), however the population were also have a problem with chronic illness even though the health care provider try to search for the new approach, it's still found that increasing mobility and mortality, especially among chronic illnesses with complications. This evidence were supported that it was lack of the appropriate intervention as well as the substantial gap of body knowledge. This research aim to implement the intervention and develop the model of QOL Improvement for chronic-patient in community by using social support.

Objective of the Study: The purposes of this study were to (1) develop the Quality Of Life (QOL) improvement for chronic illness patients in community by using social support program and (2) evaluate the effects of the program on five items; perception of illness, severity of illness, benefits and barriers of health promotion, health behaviors, QOL and stress level.

\section{MATERIALS AND METHODS}

Study design: This study was Participatory Action Research (PAR), one group pre-post test design, consisting of assessments of the data at baseline and at 3 months post intervention. Outcomes were measured on interval scales

Study Setting and participants: Cheang Yune village, a rural community, locate in Mahasarakham province, the northeast region of Thailand was selected for this intervention study.

Participants: Purposive sampling was used to select the participants. Participants were separate by 2 groups:

- The participants in the first phase compose of nurses, nutritionist, patients, caregivers, (VHVs) and research team. All of them were participate in developing the program

- The participants in the second phrase including with $10 \mathrm{VHVs}$ and 50 patients

The inclusion criteria for VHVS, being a long time residents of the community were willing to participated in this study and co-operated with the research team and health care providers, whereas the patients were volunteer, willing to participate and being able to communicate verbally.

Research instruments: Procedure for data collection relative to the intervention of chronic parents:

- Demographic data questionnaire and scales were to measure the study valuables

- The scales for measuring of the four outcome valuables were (1) perception of illness, (2) severity of illness, (3) benefits and barriers of health promotion, (4) health behaviors were developed by the researcher based on Health Believe Model (HBM) (Green and Kreuter, 1999). QOL was measured by WHOQOL-Brief-Thai (26 items) (Mahatnirunkul et al., 1998)

- $\quad$ and stress was measured by the Suanprung Stress Test-20 (Mahatnirunkul, 1997)

Interventions: A group discussion raised up for discussion to conduct the intervention base on the patients database. The participants mentioned that the appropriate program should composted of two interventions, for the VHVs and chronic illness patients.

\section{Intervention for VHVs:}

- A comprehensive 1 month program, training activities were conducted both in the community 
and hospital based. The VOV's interventions were based upon participatory planning with research team, multidisciplinary team such as primary care providers, traditional $\mathrm{RN}$ healing, nutrition therapy

- The VHVs have to learn more in the topic of chronic illness disease and self-care management, focus on DM and HT. Also, they were well trained by multidisciplinary healthcare provider team including nurse instructors, nurses, nutritionist and Thai traditional medicine team., in the topics of exercise, foot massage, health promotion activities, counseling skill, signs and symptom observation and recording, home visiting for caring the patients

Intervention for chronic illness patients: The patients received main interventions including self-care education, using traditional treatment service and home visits. The VHVs conducted a work shop for training of the patients to self-care in the topics of nutrition, exercise, foot massage and herb therapy. During work shop, the researcher team act as a mentorship and post evaluation after each session. The next step, VHVs made a home visits to visit at least once a week. They had to recording of health problems, risk behaviors and home environment follow by reported and discuss with the researcher team at least twice a week.

Data collection procedure: Procedure for data collection related to the intervention, included the completion of demographic questionnaires and scales used to measure the study variables in five study outcomes (perceive of illness, perceive of severity, perceive of beneficial and barrier, Health behaviors, Quality of life and stress level). Data collections were completed before and 2 months after the study interventions.

\section{Data analysis:}

- Descriptive statistics were used to analyze demographic data, history of illness, response to perception of illness, severity of illness, benefits and barriers of health promotion, health behaviors, QOL and stress level

- $\quad$ The paired t-test was used to test the differences of perception of illness, severity of illness, benefits and barriers of health promotion, health behaviors, QOL and stress scores between baseline and postintervention

\section{RESULTS}

Demographic characteristics: The majority of the participants were female $(n=38,76 \%)$, with the mean age of 66.68 years $(\mathrm{SD}=17.20)$, finished in primary school $(70 \%)$. Most of them caring by their children (85 and $42.5 \%$ taken care by siblings or relatives. Some participants came from low income family (40\%).

Outcomes of the Intervention: At baseline, the mean scores on each item of five study outcomes indicators (perception of severity, perception of beneficial and barrier, health behaviors, QOL and stress) were 32.16, $31.00,31.04,19.80,66.28$ and 77.86 , respectively. In contrast, the post test mean scores on the same items were: $32.86,31.52,40.00,23.20,66.00$ and 89.08 respectively.

The differences of the all variables scores between pre and post test were tested by the paired t-test. The resulted showed that after intervention, the scores of perception of benefits and barriers of health promotion health behaviors and stress level score showed a significant different. $(\mathrm{p}<0.05)$. There were not significantly different in the scores of perception of illness, severity of illness and QOL ( $\mathrm{p}>0.05)$.

\section{DISCUSSION}

The results of the study showed that the intervention was developed by multi interdisciplinary team including with patients and caregiver was increasing in perception of benefits and barriers of health promotion and health behaviors and decrease stress. The participants involved in the program and learn more about benefit of self-care including exercise, nutrition, herb therapy and home visiting by VHVs for caring, counseling, adjust the inappropriate behaviors and helping them to solve the problem, therefore, the perception of benefits and barriers and health behaviors increased in the others hand the stressful level was increase due to the social support by the VHVs, care giver and researcher team.

Although, without significant of increasing score of the perception of illness, perception of severity of illness and QOL, but from the base line data, it was represented for a high level. In this case it could be explain that the patients were concern in their illness and severity before intervention, supported by post intervention score were increasing.

Beckham et al. (2008) indicated that management of chronic illness such as diabetes mellitus is a multifaceted disease. Disease management requires a solid knowledge base combined with conscientious lifestyle change. Healthcare providers are one part of social support to encourage and help patients across their own problem by managing their own live, using innovation and culturally-specific interventions. As the same 
concept of Greenhalgh et al. (2009) who suggested, the deliberately challenged the accepted view of patient and public involvement in preventing and managing chronic illness. Whereas, Lau (2002) purposes that it is a time to shift the balance of power to include patients and their caregivers. Patient empowerment by the health care team helps patients to make judgments about their own illness "Patients are as experts of their illness and health care professionals are as experts on the medical intervention" so combining both patients and health care professional and sharing knowledge could success for disease management.

\section{CONCLUSION}

The findings suggested that this type of approach was an effective way to reach this population. Improve health care providers' knowledge and skills and increase collaborations among multidisciplinary team and Local Administration Organization (LAO), VHVs and Health Care Centre were strongly recommended in working with chronic patients. For Further study, we suggested to use PAR combine with an articulately interventions for chronically ill-patients's QOL improvement.

\section{ACKNOWLEDGEMENT}

We would like to acknowledge National Research Council of Thailand to support this project and also special thanks to Dr. Wilaiporn Rojjanasrirat Ph.D., Clinical Assistant Professor; University of Kansas, School of Nursing, Kansas City, Kansas State, USA and Dr. Apinya Wongpiriyayothar, Ph.D., RN, Faculty of Nursing, Mahasarakham University for initiate approving this article.

\section{REFERENCES}

Aujoulat, I., R. Marcolongo, L. Bonadiman and A. Deccache, 2008. Reconsidering patient empowerment in chronic illness: A critique of models of selfefficacy and bodily control. Soc. Sci. Med., 66: 1228-1239. PMID: 18155338

Beckham, S., S. Bradley, A. Washburn and T. Taumua, 2008. Diabetes management: Utilizing community health workers in a Hawaiian/Samoan population. J. Health Care Poor Underserv., 19: 416-427. http://www.ncbi.nlm.nih.gov/pubmed/18469413

Green, L.W. and M.W. Kreuter, 1999. Health Promotion Planning an Educational and Ecological Approach. 3rd Edn., McGraw-Hill, NY., pp: 162-63.
Greenhalgh, T., A. Collard and N. Begum, 2009. Sharing stories: 12 complex intervention for diabetes education in minority ethnic groups who do not speak English. BMJ., 330: 628. DOI:10.1136/bmj.330.7492.628

Lau, D.H., 2002. Patient empowerment-a patientcentred approach to Patient empowerment-apatient-centered approach to improve care. Hong Kong. Med. J., 8: 372-374. http://www.ncbi.nlm.nih.gov/pubmed/12376717

Mahatnirunkul, S., 1997. Suanprung Stress Test-20, SPST-20. State, Oct. 1, 2007.

Mahatnirunkul, S., W. Tuntipivatanakul and W. Pumpisanchai, 1998. Comparison of the WHOQOL-100 and the WHOQOL-BREF (26 items). J. Ment. Health Thai, 5: 4-15.

Panzarasaa, S., R. Bellazzi, C. Larizza and M. Stefanelli, 2004. A care flow management system for chronic patients. Study Health Technol. Reform, 107: 673-677. http://cmbi.bjmu.cn/news/report/2004/medinfo200 4/pdffiles/papers/4273Panzarasa.pdf

Rappaport, J. and E. Seidman, 2000. Community Psychology. 1st Edn., Kluwer Academic/Plenum, NY., pp: 215.

Santurri, L.E., 2006. Patient Empowerment: Improving the Outcomes of Chronic Diseases through SelfManagement Education. The MPHP 439 Online Text Book Case Western Reserve University, Spring, pp: 10.

United Nations Development Programm, (UNDP), 2007/2008. Human development report 2007. Report of Thailand.

http://hdrstats.undp.org/countries/data_sheets/ 